\section{nephron}

Practice
Nephron 2017;136:263-267

DOI: $10.1159 / 000447649$
Received: March 15, 2016

Accepted after revision: June 3, 2016 Published online: October 22, 2016

\title{
Kidney Injury Marker 1 and Neutrophil Gelatinase-Associated Lipocalin in Chronic Kidney Disease
}

\author{
Esmeralda Castillo-Rodriguez ${ }^{\text {a }}$ Raul Fernandez-Prado ${ }^{a}$ \\ Catalina Martin-Cleary ${ }^{a, b}$ Maria Soledad Pizarro-Sánchez ${ }^{a, b}$ \\ Maria Dolores Sanchez-Niño ${ }^{a}$ b Ana Belen Sanz ${ }^{a, b}$ \\ Beatriz Fernandez-Fernandez ${ }^{a, b} \quad$ Alberto Ortiz ${ }^{a-d}$

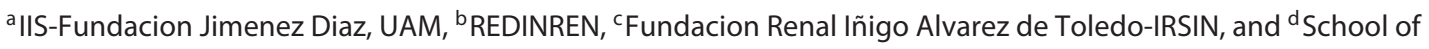 \\ Medicine, Universidad Autónoma de Madrid, Madrid, Spain
}

\section{Key Words}

Biomarker · Cardiovascular · Proximal tubular cell .

Kidney injury

\begin{abstract}
The current categorization of chronic kidney disease (CKD) is based on biomarkers of the glomerular function (estimated glomerular filtration rate, eGFR) and injury (urinary albumin creatinine ratio, UACR) and provides information on the risk of death and of progression of kidney disease. However, there are gaps in knowledge regarding the risk stratification of elderly patients with eGFR $45-60 \mathrm{ml} / \mathrm{min} / 1.73 \mathrm{~m}^{2}$ and of younger patients with higher eGFR but physiological albuminuria. In this regard, most of the kidney cell mass is composed of tubules. Recent studies have explored whether biomarkers derived from the acute kidney injury literature, which are mainly tubular injury markers, may improve the information provided by eGFR and UACR. We now review the potential role of kidney injury molecule 1 (KIM-1), hepatitis A virus cellular receptor 1, T-cell immunoglobulin and mucin domain-1 and neutrophil gelatinase-associated lipocalin (NGAL)/lipocalin 2 as biomarkers for kidney or cardiovascular outcomes in CKD patients. In general, neither uri-
\end{abstract}

\section{KARGER}

(C) 2016 S. Karger AG, Basel

E-Mail karger@karger.com

www.karger.com/nef nary KIM-1 nor urinary NGAL (UNGAL) outperform or add relevant information to eGFR or UACR. However, promising results were obtained for circulating KIM-1 prediction of renal outcomes in type 1 diabetes. Additionally, uNGAL may have some value in non-proteinuric patients and increased values have been observed in persons at risk for Mesoamerican nephropathy. Further studies are warranted in these niche populations.

(c) 2016 S. Karger AG, Basel

\section{Introduction}

Chronic kidney disease (CKD) is categorized based on biomarkers of the glomerular function (estimated glomerular filtration rate, eGFR) and injury (urinary albumin creatinine ratio, UACR) that provide information on risk of death and of progression of kidney disease. However, for certain populations, the current risk categoriza-

Contribution from the CME course of the DIABESITY Working Group of the ERA-EDTA, Bergamo, December 4-5, 2015.

E.C.-R., R.F.-P., B.F.-F. and A.O. contributed equally. 
tion may benefit from additional information regarding risk of CKD progression. As an example, kidney injury in non-albuminuric CKD (e.g. certain tubulointerstitial and vascular nephropathies) is not represented in the glomerular-based risk categorization until eGFR falls $<60 \mathrm{ml} /$ $\mathrm{min} / 1.73 \mathrm{~m}^{2}$. Tubular injury markers may be better suited for risk categorization in early stages in these patients. Kidney injury molecule 1 (KIM-1), hepatitis A virus cellular receptor 1 (HAVCR1), T-cell immunoglobulin and mucin domain 1 (TIM-1) and neutrophil gelatinase-associated lipocalin (NGAL)/lipocalin 2 (Lcn2) are tubular injury biomarkers that were among the earliest described biomarkers for acute kidney injury (AKI). Both may also add information on the persistence of renal injury in AKI and the risk of progression to CKD. We now review the potential role of KIM-1 and NGAL as biomarkers for kidney or cardiovascular outcomes in CKD patients.

\section{Kidney Injury Molecule 1}

The protein encoded by the HAVCR1 gene has received different names: KIM-1, HAVCR1 or TIM-1. We will refer to the protein as KIM-1 [1]. KIM-1 is a cell surface receptor in epithelial and lymphoid/myeloid cells. It behaves as a scavenger receptor for oxidized LDL and phosphatidylserine and as Ebola and Hepatitis A virus entry receptor in epithelial cells [2]. Kim-1 binding to phosphatidylserine allows the phagocytosis of apoptotic cells. In addition, KIM-1 regulates Th2, Th1, and Th17 differentiation. Kim-1 is markedly upregulated in proximal tubules in AKI and CKD. Indeed, the urinary excretion of the KIM-1 ectodomain was qualified by the Federal Drug Administration and by the European Medicines Agency for preclinical assessment of nephrotoxicity and on a case-by-case basis for clinical evaluation. While an acute increase in KIM-1 expression has adaptive antiinflammatory effects in experimental animals [3], chronic overexpression in proximal tubules may be maladaptive in mice and lead to MCP-1-mediated interstitial inflammation and fibrosis [4].

\section{KIM-1 (Urinary KIM-1) as a Biomarker in CKD}

A number of recent studies have evaluated the biomarker potential of urinary KIM-1 (uKIM-1) in CKD (table 1). uKIM-1 was higher in patients with proteinuria and normal renal function than in non-proteinuric individuals, and antiproteinuric drugs decreased uKIM-1, consis- tent with improved proteinuria-induced tubular injury [5]. In the Multi-Ethnic Study of Atherosclerosis (MESA) cohort of adults with normal renal function, uKIM-1 was associated with the future (5 years) risk of incident CKD stage 3 and/or rapid renal function decline, independently of UACR or NGAL levels [6]. However, the Atherosclerosis Risk in Communities Study (ARIC) evaluating nonproteinuric patients with normal renal function found no association between uKIM-1 levels and incident CKD stage 3 [7] and it did not improve the risk prediction for end-stage renal disease (ESRD) after adjusting for renal function and UACR [8]. Type 2 diabetes mellitus (T2DM) Pima Indians with macroalbuminuria had higher concentrations of uKIM-1/Cr but uKIM-1/Cr did not add to renal disease or mortality risk prediction [9]. In the Health, Aging, and Body Composition cohort, the highest quartile of uKIM- 1 was associated with heart failure risk, but the association was weaker than for UACR [10]. Furthermore, uKIM-1 had a modest association with all-cause mortality but not with cardiovascular death, whereas UACR was associated with both [11].

Circulating KIM-1 is also increased in kidney disease. In cardiopulmonary bypass, plasma KIM-1 levels increased within 2 days after surgery only in patients who developed AKI [12]. In patients with T1DM and proteinuria with normal renal function, the serum KIM-1 level at baseline strongly predicted the rate of eGFR loss and risk of ESRD after adjusting for baseline UACR, eGFR and HbAlc $[13,14]$.

\section{Neutrophil Gelatinase-Associated Lipocalin}

The Lcn2 gene encodes Lcn2, also known as NGAL. NGAL was originally identified in neutrophils, but it is a rapid response gene also expressed in kidneys and other organs in response to tissue injury. NGAL contributes to antibacterial defense by scavenging iron through binding to the siderophores that bacteria use to uptake extracellular iron [15]. The NGAL-Fe-siderophore complex is processed by tubular cells, increasing the cytoplasmic iron concentration and activating iron-dependent processes [16]. However, NGAL also binds iron through eukaryotic ligands such as catechol. Binding to NGAL decreases oxidative stress induced by the catechol-Fe complex [17]. In contrast, free NGAL may transport intracellular iron to the extracellular space [18]. Finally, NGAL promotes epithelial growth in various organs including the kidney through a yet-to-be characterized, iron-independent mechanism $[17,19]$. NGAL expression 


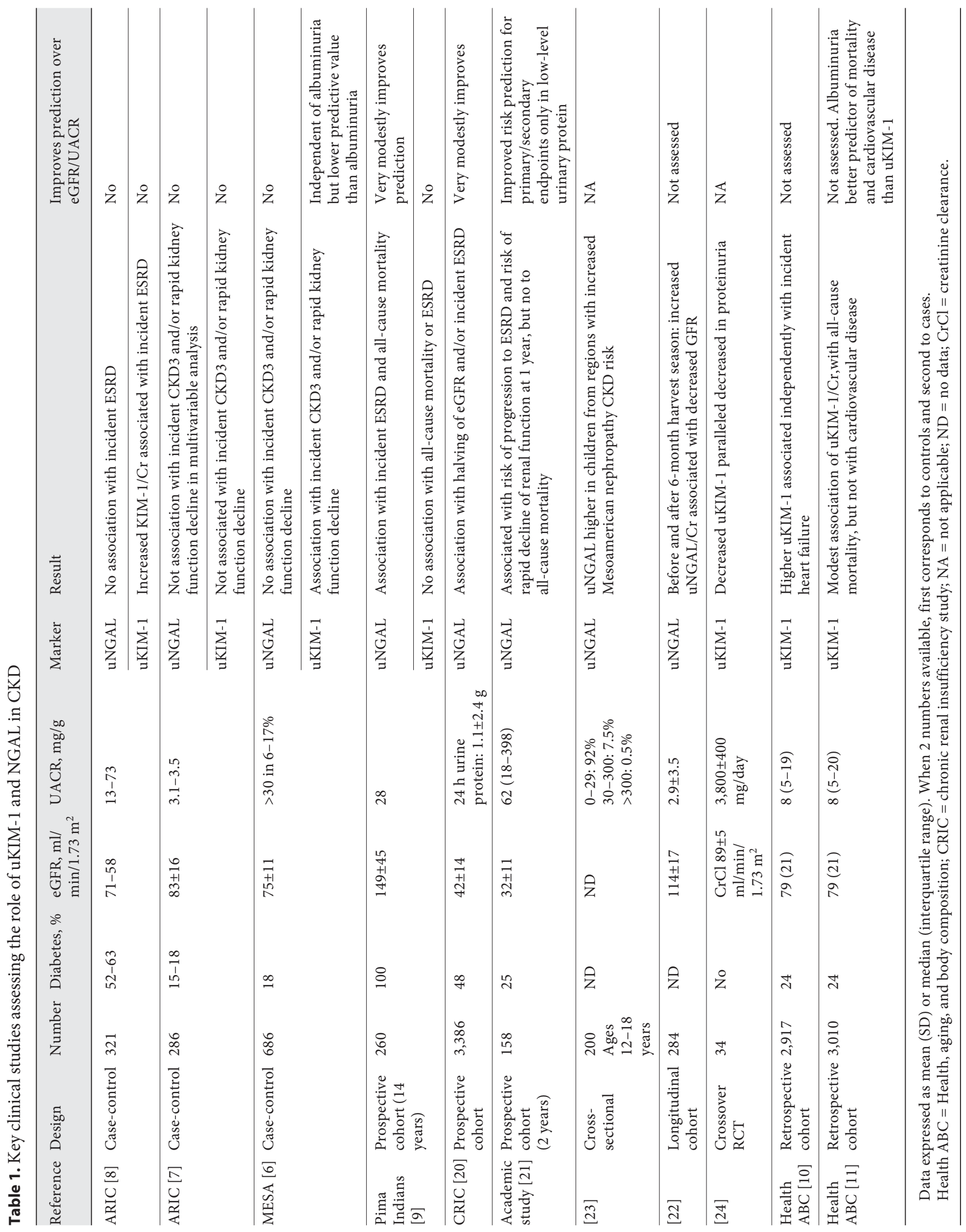


is increased during AKI, leading to high urine and serum levels in animals and patients. Most research supports a protective role for NGAL in AKI. However, in CKD progression NGAL may have a deleterious role [19].

\section{NGAL as a Biomarker in CKD}

Overall, studies have not found urinary NGAL (uNGAL) to be a strong predictor of renal outcomes in ESRD that adds relevant information on top of currently available markers. Added information, if present, was very modest (table 1). In the ARIC cohort, higher uNGAL levels were associated with incident CKD3, but significance was lost on multivariate analysis [7] and uNGAL/Cr did not associate with incident ESRD [8]. Likewise, in the MESA cohort of North American adults free of cardiovascular disease with eGFR $>60 \mathrm{ml} / \mathrm{min} / 1.73 \mathrm{~m}^{2}$, uNGAL did not associate with the risk of incident CKD3 or rapid kidney function decline at 5-year follow-up [6]. In the Chronic Renal Insufficiency Study cohort of CKD 2-4 adults, uNGAL was an independent risk factor of CKD progression but did not substantially improve outcome prediction [20]. In T2DM Pima Indians, uNGAL did not add clinically to death/ESRD risk prediction despite mild changes in C-statistics [9].

However, uNGAL may improve the prediction of CKD progression in elderly Caucasian CKD 3-4 patients with low-grade proteinuria over conventional established cardiovascular and renal risk factors [21]. Assessment of uNGAL might also provide information in Mesoamerican nephropathy, currently characterized as a chronic tubulointerstitial nephropathy. Sugarcane workers with the largest increases in $\mathrm{uNGAL} / \mathrm{Cr}$ ratios had declines in eGFR levels, measured before and after a 6-month harvest season [22], and in healthy adolescents, uNGAL but not proteinuria was increased in those regions with highest Mesoamerican nephropathy incidence [23]. Potential implications of these findings include using NGAL to identify risk factors and subclinical damage leading to Mesoamerican nephropathy.

Circulating NGAL is also increased in cardiovascular conditions, sepsis and cancer and thus is unlikely to provide information specific for CKD.

\section{Conclusions}

In conclusion, uKIM- 1 and UNGAL are markers of tubular injury and AKI that are also increased in CKD. Available studies suggest that in the CKD context, uKIM-1 or uNGAL generally do not outperform or add information to eGFR and UACR for prediction of risk of death, progression of CKD or other adverse cardiovascular outcomes. However, further research is needed on whether there might be a niche in which these biomarkers may add information, such as uNGAL for patients with normoalbuminuria or chronic tubulointerstitial nephropathy and circulating KIM-1 in diabetes.

\section{Acknowledgements}

Grant support: ISCIII and FEDER funds CP14/00133, PI13/00047, PI15/00298, Sociedad Española de Nefrologia, ISCIIIRETIC REDinREN/RD012/0021, Comunidad de Madrid CIFRA S2010/BMD-2378. Salary support: FIS Miguel Servet to M.D.S.-N., A.B.S., Joan Rodes to B.F.F. Programa Intensificación Actividad Investigadora (ISCIII/Agencia Laín-Entralgo/CM) to A.O.

\section{Disclosure Statement}

None reported.

\section{References}

1 Bonventre JV: Kidney injury molecule-1: a translational journey. Trans Am Clin Climatol Assoc 2014;125:293-299; discussion 299.

2 Kuroda M, Fujikura D, Nanbo A, et al: Interaction between TIM-1 and NPC1 is important for cellular entry of Ebola virus. J Virol 2015;89:6481-6493.

3 Yang L, Brooks CR, Xiao S, et al: KIM-1-mediated phagocytosis reduces acute injury to the kidney. J Clin Invest 2015;125:16201636 .
4 Humphreys BD, Xu F, Sabbisetti V, et al: Chronic epithelial kidney injury molecule-1 expression causes murine kidney fibrosis. J Clin Invest 2013;123:4023-4035.

5 Duruöz MT, Poiraudeau S, Fermanian J, et al: Development and validation of a rheumatoid hand functional disability scale that assesses functional handicap. J Rheumatol 1996;23: 1167-1172.

6 Peralta CA, Katz R, Bonventre JV, et al: Associations of urinary levels of kidney injury molecule 1 (KIM-1) and neutrophil gelatinase-associated lipocalin (NGAL) with kidney function decline in the multi-ethnic study of atherosclerosis (MESA). Am J Kidney Dis 2012;60:904-911.

7 Bhavsar NA, Köttgen A, Coresh J, et al: Neutrophil gelatinase-associated lipocalin (NGAL) and kidney injury molecule 1 (KIM-1) as predictors of incident CKD stage 3: the atherosclerosis risk in communities (ARIC) study. Am J Kidney Dis 2012;60:233-240.

8 Foster MC, Coresh J, Bonventre JV, et al: Urinary biomarkers and risk of ESRD in the atherosclerosis risk in communities study. Clin J Am Soc Nephrol 2015;10:1956-1963. 
9 Fufaa GD, Weil EJ, Nelson RG, et al: Association of urinary KIM-1, L-FABP, NAG and NGAL with incident end-stage renal disease and mortality in American Indians with type 2 diabetes mellitus. Diabetologia 2015;58: 188-198.

10 Driver TH, Katz R, Ix JH, et al: Urinary kidney injury molecule 1 (KIM-1) and interleukin 18 (IL-18) as risk markers for heart failure in older adults: the health, aging, and body composition (health $\mathrm{ABC}$ ) study. Am J Kidney Dis 2014;64:49-56.

11 Sarnak MJ, Katz R, Newman A, et al: Association of urinary injury biomarkers with mortality and cardiovascular events. J Am Soc Nephrol 2014;25:1545-1553.

12 Schley G, Köberle C, Manuilova E, et al: Comparison of plasma and urine biomarker performance in acute kidney injury. PLoS One 2015;10:e145042.

13 Sabbisetti VS, Waikar SS, Antoine DJ, et al: Blood kidney injury molecule- 1 is a biomarker of acute and chronic kidney injury and predicts progression to ESRD in type I diabetes. J Am Soc Nephrol 2014;25:2177-2186.
14 Nowak N, Skupien J, Niewczas MA, et al: Increased plasma kidney injury molecule-1 suggests early progressive renal decline in nonproteinuric patients with type 1 diabetes. Kidney Int 2016;89:459-467.

15 Nasioudis D, Witkin SS: Neutrophil gelatinase-associated lipocalin and innate immune responses to bacterial infections. Med Microbiol Immunol 2015;204:471-479.

16 Schmidt-Ott KM, Mori K, Li JY, et al: Dual action of neutrophil gelatinase-associated lipocalin. J Am Soc Nephrol 2007;18:407-413.

17 Paragas N, Qiu A, Hollmen M, et al: NGALsiderocalin in kidney disease. Biochim Biophys Acta 2012;1823:1451-1458.

18 Devireddy LR, Gazin C, Zhu X, et al: A cellsurface receptor for lipocalin $24 \mathrm{p} 3$ selectively mediates apoptosis and iron uptake. Cell 2005; 123:1293-1305.

19 Viau A, El Karoui K, Laouari D, et al: Lipocalin 2 is essential for chronic kidney disease progression in mice and humans. J Clin Invest 2010;120:4065-4076.

20 Liu KD, Yang W, Anderson AH, et al: Urine neutrophil gelatinase-associated lipocalin levels do not improve risk prediction of progressive chronic kidney disease. Kidney Int 2013;83:909-914.
21 Smith ER, Lee D, Cai MM, et al: Urinary neutrophil gelatinase-associated lipocalin may aid prediction of renal decline in patients with non-proteinuric stages 3 and 4 chronic kidney disease (CKD). Nephrol Dial Transplant 2013;28:1569-1579.

22 Laws RL, Brooks DR, Amador JJ, et al: Biomarkers of kidney injury among Nicaraguan sugarcane workers. Am J Kidney Dis 2016;67: 209-217.

23 Ramírez-Rubio O, Amador JJ, Kaufman JS, et al: Urine biomarkers of kidney injury among adolescents in Nicaragua, a region affected by an epidemic of chronic kidney disease of unknown aetiology. Nephrol Dial Transplant 2016;31:424-432.

24 Waanders F, Vaidya VS, van Goor H, et al: Effect of renin-angiotensin-aldosterone system inhibition, dietary sodium restriction, and/or diuretics on urinary kidney injury molecule 1 excretion in nondiabetic proteinuric kidney disease: a post hoc analysis of a randomized controlled trial. Am J Kidney Dis 2009;53:16-25. 